\title{
Growth performance, body composition and fatty acid profile of liver and muscle in tilapia, Oreochromis mossambicus (Peters, 1852) fed algal meal incorporated diets
}

\author{
S. K. PATRA, K. MISHRA, B. PATRO, K. SAMANTARAY, B. K. KHUNTIA, B. SAHU \\ AND M. K. TRIPATHY \\ College of Fisheries, Orissa Univeristy of Agriculture and Technology, Rangeilunda, Berhampur - 760007 \\ Odisha, India \\ e-mail:ksamantaray@yahoo.com
}

\begin{abstract}
The present study was conducted to evaluate the effect of algal meal incorporated diets on the growth performance and fatty acid profile of tilapia Oreochromis mossambicus (Peters, 1852). Control diet (Diet 1) for the study was formulated with rice bran, groundnut oil cake (GNOC) and soybean meal. Six iso-energetic and iso-nitrogenous test diets (Diets 2 to 7) were prepared by incorporating (@40\%) one of the four microalgae viz., Anabaena cylindrical (AN; Nostoc salbasa (NS); Spirulina platensis (SP) and Westiellopsis prolifica (WS) or with an algal meal mixture containing the above four algae plus the macroalgal species viz., Gracilaria edulis (GE) and Enteromorpha intestinalis (EI) mixed in equal proportions along with sunflower oil and cod liver oil (by totally replacing rice bran, GNOC and soyabean meal of the control diet) (AMM $+\mathrm{O}$ diet, Diet 6) and AMM without addition of oils served as AMM diet (Diet 7). The algal meal supplemented diets were tested against a control diet to find out their suitability for culture of tilapia fingerlings. The fish (av. wt. $2.8 \pm 0.4 \mathrm{~g}$ ) were fed up to satiation for 56 days at $28 \pm 2^{\circ} \mathrm{C}$. Significant $(\mathrm{p}<0.05)$ enhancement in growth was observed with AN $(565.2 \%)$ diet followed by SP (500\%) and WS (400\%) compared to control (402.3\%). The AN diet showed best performance in terms of feed conversion ratio (FCR), protein efficiency ratio (PER) and specific growth rate (SGR) while AMM diet showed least efficiency. Fish fed with SP diet showed better apparent net protein utilisation (ANPU\%) as compared to other diets. Saturated fatty acid (SFA) in the liver and muscle decreased while monounsaturated (MUFA) and polyunsaturated (PUFA) fatty acids increased over their respective initial values in all the dietary groups except in groups fed with AMM $+\mathrm{O}$ and AMM. The n-6 level of liver and muscle increased with SP diets. Substantial increase in docosahexaenoic acid (DHA, 22:6n3) and eicosapentaenoic acid (EPA, 20:5n3) in fish fed with WS emphasised the benefits of its use as dietary ingredients for tilapia. The results clearly indicated that algae like AN and SP incorporated in tilapia diet significantly enhance growth and carcass protein, whereas WS helps to improve the levels of the essential n-3 fatty acids (EPA and DHA).
\end{abstract}

Keywords: Algal meal diets, Fatty acid profile, Growth, Oreochromis mossambicus, Tilapia

\section{Introduction}

Tilapia is the second most important group of farmed fish after carps worldwide. Global tilapia production estimated at 4.5 million $\mathrm{t}$ in 2012, was predicted to exceed 4.8 million $\mathrm{t}$ in 2014 (@6\% growth) (FAO, 2014). Their hardiness and adaptability to a wide range of culture systems has led to the commercialisation of tilapia production in more than 100 countries. In comparison to freshwater fishes, the popularity of marine species as a healthy food commodity has increased tremendously, due to their higher content of n-3 fatty acids like eicosapentaenoic acid (EPA) (20:5n3) and docosahexaenoic acid (DHA) (22:6n3). In marine environment, fish consume marine algae that are rich in n-3 fatty acids. It has been reported that dietary modulations can improve the levels of such fatty acids in the tissues of freshwater fish, so as to provide healthy diet for human beings (Steffens, 1997). The growth of fish depends both on protein and lipid levels as lipid has protein sparing effect. Fish mobilises saturated and monoenoic fatty acid as source of energy and retains polyunsaturated fatty acids (PUFA) in the body (Steffens, 1997). It has been confirmed that inclusion of algae in the diet significantly improves growth rate in red sea bream (Mustafa et al., 1995) and common carp (Oramary et al., 2016). On the other hand, the deficiency of dietary PUFA has been reported to affect body compositional changes in fish (Lund et al., 2014).

Growth performance and lipid metabolism was studied in Atlantic salmon (Salmo salar) using two algae derived products Verdemin (derived from Ulva ohnoi) and Rosamin (derived from diatom Entomoneis spp.) replacing fish meal (Norambuena et al., 2015). Hepher et al. (1978) pointed out that as methods of algal culture and harvesting 
are improved, the economic provision of algal meals as dietary protein and lipid sources become more probable. Importantly, commercial culture methods of algae, using low cost technology, have been established for various uses (Padhi, 1983; Patanaik et al., 1995). Therefore, algae are used as primary source of food for the larvae and juveniles of several bivalves, crustaceans and finfishes in mariculture (Brown et al., 1997). There have been a number of studies on use of algal meal as a dietary source of nutrient for several marine fishes (Matty and Smith, 1978; Appler, 1985). Several research works carried on marine fish species have confirmed that the dietary levels directly influence the fatty acid profile of fish (Stickney and Hardy, 1989; Brown et al., 1997). However, very little information is available on the same aspect in case of freshwater fish species (De Silva et al., 1997; Mishra, 2000; Mishra and Samantaray, 2004).

Most of the tropical freshwater fish species used in aquaculture including tilapia have lower content of n-3 fatty acids (Devadasan, 2002). Hence, the present investigation aimed at enhancing the growth performance and improving the fatty acid profile particularly of n-3 fatty acids (EPA and DHA) in tilapia Oreochromis mossambicus (Peters, 1852), using algal meal incorporated diets.

\section{Materials and methods}

\section{Algal meal}

Four species of freshwater microalgae viz., Anabaena cylindrical (AN), Nostoc salbasa (NS), Spirulina platensis (SP), Westiellopsis prolifica (WS) and two species of macro algae, Gracilaria edulis (GE) and Enteromorpha intestinalis (EI) isolated and identified by experts of the Algal Research Laboratory, Berhampur University, Odisha were used in the present study. The algae were cultured and harvested during the log phase as reported earlier (Mishra and Samantaray, 2000), washed thoroughly, centrifuged at $4000 \mathrm{~g}$, hand pressed and on blotting paper to remove excess water. Then the algae were dried in an oven for $15 \mathrm{~min}$ at $60^{\circ} \mathrm{C}$ followed by air drying at room temperature for $45 \mathrm{~min}$ and ground to get the algal meal. The algal meals were kept in sealed polythene bags inside refrigerator until further use. The proximate composition and fatty acid profile of different algae, used in this study for diet formulation, has already been reported earlier (Mishra, 2000; Mishra and Samantaray, 2000).

\section{Experimental diets}

Six iso-energetic (14.73 MJ gross energy $\mathrm{kg}^{-1}$ of diet) and iso-nitrogenous (32\% crude protein) (Mishra and Samantaray, 2004) experimental diets containing algae as the major dietary ingredient were tested against a control diet. Rice bran (crude protein 12-13\%, crude fat $5 \%$ ), groundnut oil cake (GNOC) (crude protein 44.2\%, crude fat $10 \%$ ) and soybean meal (crude protein $46.3 \%$, crude fat $1.3 \%$ ) were used to formulate the control diet (Diet 1) (Table 1). In diet 2 to 5, 40\% of the ingredients of the control diet were replaced by algal meal prepared from one of the four microalgae, namely AN (Diet 2), NS (Diet 3), SP (Diet 4) and WS (Diet 5), that were found to be rich in protein and lipid (Mishra, 2000). Sunflower oil (Agro Tech Foods Ltd., India) and cod liver oil (Seven Seas, Ireland, UK) were added at a level of $1 \%$ to each of the diet 1 to 5 as the source of lipid. GE and EI along with the above four algae were mixed in equal proportions to prepare an algal meal mixture by totally replacing rice bran, GNOC and soyabean meal of the control diet. The algal meal mixture along with above oils (sunflower oil and cod liver oil) was used to make AMM+O diet (Diet 6). The diet prepared with algal meal mixture (by using above six algae in equal proportion as described previously), without addition of oils (sunflower oil and cod liver oil) was used as AMM diet (Diet 7). aCellulose and carboxymethyl cellulose (CMC) (Analar Grade, $\mathrm{CDH}$, India) at $1 \%$ level and vitamin and mineral mixture (Roche India Ltd.) at $2 \%$ level were used in all the diets.

The required ingredients for each diet were ground in an electrical grinder along with $0.05 \mathrm{~g} \mathrm{~kg}^{-1}$ butylated hydroxyl toluene (BHT) before the dough was prepared. The pellets were prepared as described by Samantaray and Mohanty (1997) and were stored in refrigerator in airtight containers having nitrogen gas. The diets were prepared on weekly ration basis in order to avoid nutrient loss.

\section{Experimental design}

Tilapia, O. mossambicus fingerlings measuring $4.1 \pm 0.6 \mathrm{~cm}$ in length and $2.8 \pm 0.4 \mathrm{~g}$ in weight were randomly stocked (@12 to 14 nos.per tank) to have a total biomass of $30 \pm 2 \mathrm{~g}$ in each of the 14 nos. of 651 capacity FRP tanks under a flow through system. The flow rate of $90 \mathrm{ml} \mathrm{min}$ mas $^{-1}$ wa maintained in the flow through tanks. The experiment was conducted using the seven diets (Diet 1 to 7 ) in duplicates for 56 days. Prior to initiation of the experiment, fish were acclimated for 2 weeks to the experimental conditions by feeding a diet in which $20 \%$ of the ingredients of the control diet (diet 1) were replaced by algal meal mixture. Supplemental aeration was provided in the tanks for $8 \mathrm{~h}$ a day (08 00 to $1600 \mathrm{hrs)}$ on regular basis for better feed utilisation. Water temperature was maintained at $28 \pm 2^{\circ} \mathrm{C}$ by using thermostatically controlled electric aquarium heater. Dissolved oxygen and $\mathrm{pH}$ in the experimental tanks were recorded regularly for the entire experimental period and was found to vary between 6 to $8 \mathrm{ppm}$ and 7.7 to 8.1 , respectively. A 12:12 h light and dark cycle was maintained by incandescent lighting for the entire period of the experiment. The fishes were hand fed up to satiation twice daily at 0900 and $1500 \mathrm{hrs}, 7$ days a week, throughout the experiment. The uneaten feed, if any, were removed from each tank separately after $2 \mathrm{~h}$ of feeding, oven-dried and weighed to record actual 
Table 1. Ingredients and nutrient composition of the test diets (\% dry matter basis)

\begin{tabular}{|c|c|c|c|c|c|c|c|}
\hline \multirow{2}{*}{ Ingredients } & \multicolumn{6}{|c|}{ Dietary code ${ }^{*}$} & \multirow[b]{2}{*}{ AMN } \\
\hline & $\mathrm{C}$ & AN & NS & SP & WS & $\mathrm{AMM}+\mathrm{O}$ & \\
\hline Algae & - & 40 & 40 & 40 & 40 & 94 & 96 \\
\hline Rice bran ${ }^{1}$ & 25.4 & - & - & 27.0 & 27.0 & - & - \\
\hline GNOC $^{1}$ & 33.5 & 27.0 & 27.0 & 27.0 & 27.0 & - & - \\
\hline Soyabean meal ${ }^{1}$ & 35.1 & 27.0 & 27.0 & - & - & - & - \\
\hline Sunflower oil ${ }^{2}$ & 1.0 & 1.0 & 1.0 & 1.0 & 1.0 & 1.0 & - \\
\hline Cod liver oil ${ }^{3}$ & 1.0 & 1.0 & 1.0 & 1.0 & 1.0 & 1.0 & - \\
\hline$\alpha$-Cellulose $e^{4}$ & 1.0 & 1.0 & 1.0 & 1.0 & 1.0 & 1.0 & 1.0 \\
\hline Carboxymethyl cellulose ${ }^{4}$ & 1.0 & 1.0 & 1.0 & 1.0 & 1.0 & 1.0 & 1.0 \\
\hline Vitamin and mineral mixture ${ }^{5}$ & 2.0 & 2.0 & 2.0 & 2.0 & 2.0 & 2.0 & 2.0 \\
\hline \multicolumn{8}{|l|}{ Nutrient content } \\
\hline Moisture & 7.7 & 7.8 & 8.2 & 8.0 & 8.1 & 7.9 & 8.4 \\
\hline Crude protein & 32.4 & 33.6 & 33.0 & 32.4 & 31.4 & 31.3 & 32.3 \\
\hline Crude lipid & 8.8 & 6.0 & 6.0 & 8.51 & 8.7 & 5.2 & 4.4 \\
\hline Gross energy $\left(\mathrm{MJ} \mathrm{kg}^{-1}\right)$ & 14.81 & 14.69 & 14.85 & 14.89 & 14.60 & 14.81 & 14.56 \\
\hline $\mathrm{P} / \mathrm{E}$ ratio (mg protein/kcal energy) & 91.5 & 95.73 & 93.0 & 91.0 & 89.5 & 88.4 & 93.0 \\
\hline
\end{tabular}

"Control, C (Diet 1); Anabaena cylindrical, AN (Diet 2); Nostoc salbasa, NS (Diet 3); Spirulina platensis, SP (Diet 4); Westiellopsis prolifica WS (Diet 5); Algal meal mixture with supplemental oil, AMM+O (Diet 6); Algal meal mixture, AMM (Diet 7)

${ }^{1}$ Locally available ingredients

${ }^{2}$ Agro Tech Foods Ltd., Secunderabad, India

${ }^{3}$ Seven Seas Make, Ireland, UK

${ }^{4}$ Analar Grade, $\mathrm{CDH}$, Mumbai, India

${ }^{5}$ Roche India Ltd., Mumbai, India. Composition of the mixture to supply per $100 \mathrm{~g}$ dry diet: Vitamins : vitamin A (as acetate), 10000 IU; cholecalciferol (Vit. D3), $1000 \mathrm{IU}$; thiamin mononitrate, $10 \mathrm{mg}$; riboflavin, $10 \mathrm{mg}$; pyridoxine hydrochloride, 60mg; cyanocobalamin,30 $\mu \mathrm{g}$; nicotinamide, $200 \mathrm{mg}$; ascorbic acid, $300 \mathrm{mg}$; $\alpha$-tocopheryl acetate, $50 \mathrm{mg}$; biotin, $0.50 \mathrm{mg}$. Minerals : calcium phosphate, $258 \mathrm{mg}$; magnesium oxide light, $120 \mathrm{mg}$; dried ferrous sulphate $64.08 \mathrm{mg}$; manganese sulphate, $4.06 \mathrm{mg}$; Total phosphorous in the preparation, $50.16 \mathrm{mg}$. Trace elements: copper sulphate, $6.78 \mathrm{mg}$; zinc sulphate, $4.40 \mathrm{mg}$; sodium molybdate, $0.50 \mathrm{mg}$; sodium borate, $1.76 \mathrm{mg}$

feed consumption. Fish were weighed individually at the beginning and at the end of the experiment to record the growth parameters but batch weighing was done at 15 day interval for feed adjustment.

To study the nutrient digestibility, in the last week of the experiment, $1 \%$ of cellulose was replaced by chromium oxide which was used as an external marker in the diet. The faecal matter collected from each tank every day before first feeding was dried and pooled together. The chromium oxide in the feed and faecal matter was estimated as per the method of Furukawa and Tsukahara (1966).

Twelve representative samples of fish were taken at the beginning of the experiment to record the initial carcass composition as well as the liver and dorsal muscle fatty acid profile. At the end of the experiment 6 nos. of fish from each tank were used for final carcass analysis (AOAC, 1975) and fatty acid analysis of dorsal muscle and liver.

\section{Growth analysis}

Growth in terms of weight gain (\%), average daily weight gain (\% ADG), specific growth rate (\%SGR), feed conversion ratio (FCR), protein efficiency ratio (PER) and apparent net protein utilisation (\% ANPU) were calculated as per standard formulae (Samantaray and Mohanty, 1997).

\section{Proximate composition analysis}

Algal protein was analysed according to the microburette method given by Rausch (1981) and Meyer and Walther (1988). The algal samples were extracted with chloroform methanol mixture $(2: 1)$ containing $0.05 \mathrm{~g} \mathrm{~kg}^{-1}$ butylated hydroxytoluene (BHT) (Boberg et al., 1985). The homogenate was added with $15 \mathrm{ml}$ of $0.2 \mathrm{M}$ sodium dihydrogen phosphate. After thorough mixing, the non-aqueous chloroform layer was pipetted off, evaporated and weighed to determine algal lipid.

Experimental diets and fish carcass were analysed for moisture, crude protein, crude fat and ash content following AOAC (1975) method. The gross energy content of the diets was measured in Reico plain oxygen bomb calorimeter (Samantaray and Mohanty, 1997). The chromium oxide in the diet was determined spectrophotometrically and the apparent digestibility of the protein and lipid was calculated as per the method of Furukawa and Tsukahara (1966).

\section{Fatty acid (FA) analysis}

The total lipids of the samples (algae, diets and fish tissues) were extracted as described earlier for algae. The FAs in the lipid extract were transmethylated at $60^{\circ} \mathrm{C}$ overnight 
after addition of $2 \mathrm{ml}$ of $5 \% \mathrm{H}_{2} \mathrm{SO}_{4}$ in methanol (Boberg et al., 1985). The solvent phase was extracted as described by Mishra and Samantaray (2004) and evaporated in $1 \mathrm{ml}$ hexane to which a known amount $(25 \mathrm{mg})$ of an internal standard (22:0) was added and analysed by injecting fixed quantity in a HP 5890 Gas Chromatograph equipped with 25 mmegabore $(530 \mu \mathrm{m})$ methyl silicon fused column with film thickness $2.65 \mathrm{~mm}$ (Hewlett-Packard-I) and an FID detector. Nitrogen was used as a carrier gas at a regulated pressure of $20 \mathrm{ml} \mathrm{min}{ }^{-1}$. The run method was through a temperature gradient of $150-250^{\circ} \mathrm{C}$ with injector and detector temperature at 200 and $250^{\circ} \mathrm{C}$, respectively.

The individual FAs were identified by comparing the retention times with commercially available reference standards of FA mixture (Supelco 37, component, Supelco, USA) and individual FA standard (Sigma, USA) as described by Gopakumar and Nair (1972) and comparing the area of peaks with the peak of internal standard (22:0) (Ahlgren, 1992). Fatty acids whose standards were unavailable could not be identified and mentioned as others. The FA compositions of experimental diets are shown in Table 2.

\section{Statistical analysis}

Data were analysed using analysis of variance (ANOVA). Duncan's Multiple Range Test (Duncan, 1955) was used to evaluate the mean difference among experimental groups at 5\% level of significance. All the statistical analyses were done in an IBM compatible PC-AT using SPSS (ver.10).

\section{Results}

No mortality was observed during the entire study period. The effects of experimental diets on the growth performance and nutrient digestibility are shown in Table 3. The control diet was effectively utilised by the tilapia fingerlings in terms of weight gain $(402.3 \%)$ and growth performance. Tilapia

Table 2. Fatty acid profile (\% of the total fatty acid) of the experimental diets

\begin{tabular}{|c|c|c|c|c|c|c|c|}
\hline Fatty acid & Control & AN & NS & $\mathrm{SP}$ & WS & $\mathrm{AMM}+\mathrm{O}$ & AMM \\
\hline $12: 0$ & $6.1 \pm 0.4$ & $8.6 \pm 0.4$ & $6.1 \pm 0.2$ & $8.3 \pm 0.1$ & $6.2 \pm 0.3$ & $7.7 \pm 0.2$ & $8.6 \pm 0.1$ \\
\hline $14: 0$ & $10.6 \pm 0.1$ & $12.4 \pm 0.3$ & $12.3 \pm 0.2$ & $13.0 \pm 0.2$ & $14.4 \pm 0.2$ & $14.4 \pm 0.2$ & $16.4 \pm 0.2$ \\
\hline $14: 1$ & $3.2 \pm 0.01$ & $7.2 \pm 0.2$ & $6.1 \pm 0.2$ & $6.2 \pm 0.1$ & $6.3 \pm 0.04$ & $5.3 \pm 0.1$ & $8.3 \pm 0.2$ \\
\hline $15: 1$ & $2.5 \pm 0.01$ & $3.3 \pm 0.01$ & $4.1 \pm 0.02$ & $3.2 \pm 0.02$ & - & $1.1 \pm 0.01$ & $2.3 \pm 0.01$ \\
\hline $16: 0$ & $16.9 \pm 0.2$ & $17.5 \pm 0.2$ & $16.9 \pm 0.2$ & $17.8 \pm 0.2$ & $14.4 \pm 0.2$ & $14.9 \pm 0.2$ & $19.9 \pm 0.1$ \\
\hline $16: 1$ & $6.2 \pm 0.1$ & $5.3 \pm 0.1$ & $6.1 \pm 0.01$ & $5.6 \pm 0.01$ & $3.3 \pm 0.01$ & $5.2 \pm 0.01$ & $2.3 \pm 0.02$ \\
\hline $18: 0$ & $14.0 \pm 0.4$ & $12.9 \pm 0.1$ & $13.2 \pm 0.2$ & $12.0 \pm 0.1$ & $15.6 \pm 0.2$ & $12.3 \pm 0.1$ & $15.8 \pm 0.3$ \\
\hline $18: \ln 7$ & $7.8 \pm 0.2$ & $5.2 \pm 0.1$ & $4.3 \pm 0.1$ & $3.1 \pm 0.03$ & $2.6 \pm 0.01$ & $7.2 \pm 0.06$ & $5.4 \pm 0.1$ \\
\hline $18: \ln 9$ & $8.3 \pm 0.1$ & $8.0 \pm 0.1$ & $7.0 \pm 0.1$ & $6.9 \pm 0.2$ & $9.3 \pm 0.3$ & $7.2 \pm 0.2$ & $6.3 \pm 0.1$ \\
\hline $18: 2 \mathrm{n} 6$ & $4.0 \pm 0.1$ & $5.3 \pm 0.1$ & $4.8 \pm 0.2$ & $6.6 \pm 0.4$ & $4.6 \pm 0.3$ & $6.3 \pm 0.2$ & $2.3 \pm 0.01$ \\
\hline $18: 3 n 6$ & - & - & - & $2.5 \pm 0.02$ & - & $1.0 \pm 0.01$ & $1.0 \pm 0.01$ \\
\hline $18: 3 n 3$ & $2.7 \pm 0.01$ & $2.5 \pm 0.02$ & $3.4 \pm 0.02$ & - & $6.0 \pm 0.1$ & $3.9 \pm 0.02$ & - \\
\hline $20: 0$ & 0.6 & $1.7 \pm 0.01$ & $1.1 \pm 0.01$ & $1.2 \pm 0.01$ & 0.7 & $2.6 \pm 0.01$ & $3.0 \pm 0.02$ \\
\hline $20: 2$ n6 & $4.4 \pm 0.02$ & $2.3 \pm 0.02$ & $5.3 \pm 0.1$ & $2.1 \pm 0.01$ & $5.2 \pm 0.04$ & $3.3 \pm 0.03$ & - \\
\hline $20: 3 n 6$ & $2.8 \pm 0.02$ & $1.1 \pm 0.04$ & - & $2.3 \pm 0.06$ & $2.6 \pm 0.01$ & 0.9 & 0.8 \\
\hline $20: 3 n 3$ & $1.1 \pm 0.03$ & $1.0 \pm 0.01$ & $1.5 \pm 0.02$ & $1.1 \pm 0.01$ & $2.2 \pm 0.04$ & 0.6 & - \\
\hline $20: 4 n 6$ & $2.1 \pm 0.03$ & $1.3 \pm 0.01$ & $1.2 \pm 0.01$ & $1.9 \pm 0.02$ & $2.0 \pm 0.03$ & - & - \\
\hline $20: 5 n 3$ & $1.1 \pm 0.01$ & $1.3 \pm 0.01$ & $1.4 \pm 0.01$ & - & $2.1 \pm 0.02$ & 0.6 & - \\
\hline 22:0 & $2.1 \pm 0.01$ & 1.0 & 0.6 & - & - & $1.9 \pm 0.01$ & $2.89 \pm 0.03$ \\
\hline $22: 6 n 3$ & - & - & - & - & 0.5 & - & - \\
\hline $24: \ln 9$ & - & - & - & $2.6 \pm 0.03$ & - & - & - \\
\hline FA & $96.5 \pm 2.3$ & $96.6 \pm 2.6$ & $95.4 \pm 2.2$ & $96.4 \pm 2.1$ & $97.5 \pm 1.9$ & $96.4 \pm 2.0$ & $95.3 \pm 2.3$ \\
\hline Others & $3.5 \pm 0.06$ & $3.4 \pm 0.08$ & $4.6 \pm 0.06$ & $3.6 \pm 0.04$ & $2.5 \pm 0.01$ & $3.6 \pm 0.01$ & $4.7 \pm 0.01$ \\
\hline$\Sigma$ SFA & $50.3^{b} \pm 1.0$ & $54.1^{b} \pm 1.1$ & $50.2^{\mathrm{b}} \pm 1.2$ & $52.3^{\mathrm{b}} \pm 0.9$ & $51.3^{\mathrm{b}} \pm 0.5$ & $53.8^{\mathrm{b}} \pm 0.6$ & $66.6^{a} \pm 0.5$ \\
\hline$\Sigma$ MUFA & $28.0^{\mathrm{a}} \pm 0.3$ & $29.0^{\mathrm{a}} \pm 0.2$ & $27.6^{\mathrm{ab}} \pm 0.2$ & $27.6^{\mathrm{ab}} \pm 0.3$ & $21.5^{\mathrm{d}} \pm 0.2$ & $26.0^{\mathrm{bc}} \pm 0.3$ & $24.6^{\mathrm{bc}} \pm 0.4$ \\
\hline$\Sigma$ PUFA & $18.2^{\mathrm{b}} \pm 0.2$ & $14.8^{\mathrm{c}} \pm 0.2$ & $17.6^{\mathrm{bc}} \pm 0.2$ & $16.5^{b c} \pm 0.2$ & $25.2^{\mathrm{a}} \pm 0.2$ & $16.6^{\mathrm{bc}} \pm 0.3$ & $4.1^{\mathrm{d}} \pm 0.01$ \\
\hline$\Sigma \mathrm{n} 3$ & $4.9^{d} \pm 0.06$ & $4.8^{\mathrm{d}} \pm 0.03$ & $6.3^{b} \pm 0.1$ & $1.1^{\mathrm{d}} \pm 0.02$ & $10.3^{a} \pm 0.1$ & $5.1^{c} \pm 0.1$ & - \\
\hline$\Sigma \mathrm{n} 6$ & $13.3^{c} \pm 0.1$ & $10.0^{\mathrm{e}} \pm 0.1$ & $11.3^{\mathrm{d}} \pm 0.1$ & $15.4^{\mathrm{a}} \pm 0.2$ & $14.4^{\mathrm{b}} \pm 0.1$ & $11.5^{\mathrm{d}} \pm 0.1$ & $4.1^{\mathrm{f}} \pm 0.06$ \\
\hline $\mathrm{n} 3 / \mathrm{n} 6$ & $0.37^{\mathrm{d}}$ & $0.48^{c}$ & $0.58^{\mathrm{b}}$ & $0.07^{\mathrm{e}}$ & $0.71^{\mathrm{a}}$ & $0.44^{\mathrm{c}}$ & 0.0 \\
\hline
\end{tabular}

Data presented as Mean \pm SE $(n=6)$

Mean values for components with the same superscripts within the row are not significantly different $(\mathrm{p}>0.05)$ 
Table 3. Growth performance and nutrient digestibility of tilapia fingerlings fed with the experimental diets for 56 days

\begin{tabular}{llllllll}
\hline Parameters & $\mathrm{C}$ & $\mathrm{AN}$ & $\mathrm{NS}$ & $\mathrm{SP}$ & WS & AMM $+\mathrm{O}$ & AMM \\
\hline Initial weight (g) & $2.8 \pm 0.06$ & $2.5 \pm 0.05$ & $3.1 \pm 0.05$ & $2.9 \pm 0.03$ & $2.8^{2} \pm 0.03$ & $2.4 \pm 0.02$ & $2.5 \pm 0.04$ \\
Final weight (g) & $14.1^{\mathrm{b}} \pm 0.3$ & $16.6^{\mathrm{b}} \pm 0.4$ & $13.9^{\mathrm{b}} \pm 0.4$ & $17.4^{\mathrm{a}} \pm 0.4$ & $14.0^{\mathrm{b}} \pm 0.2$ & $9.8^{\mathrm{c}} \pm 0.2$ & $9.9^{\mathrm{c}} \pm 0.2$ \\
Weight gain (\%) & $402.3^{\mathrm{c}} \pm 6.1$ & $565.2^{\mathrm{a}} \pm 6.9$ & $348.4^{\mathrm{d}} \pm 4.1$ & $500.0^{\mathrm{b}} \pm 5.3$ & $400.0^{\mathrm{c}} \pm 4.4$ & $308.3^{\mathrm{e}} \pm 3.8$ & $297.2^{\mathrm{f}} \pm 4.0$ \\
Feed intake (g) & $15.8 \pm 0.9$ & $18.0 \pm 0.8$ & $17.6 \pm 1.0$ & $21.4 \pm 1.0$ & $15.9 \pm 0.7$ & $14.1 \pm 0.7$ & $16.3 \pm 0.8$ \\
Protein intake (g) & $5.1 \pm 0.1$ & $6.1 \pm 0.3$ & $5.8 \pm 0.3$ & $6.9 \pm 0.2$ & $4.9 \pm 0.1$ & $4.4 \pm 0.1$ & $5.2 \pm 0.3$ \\
ADG (\%) & $20.0^{\mathrm{b}} \pm 0.4$ & $25.2^{\mathrm{a}} \pm 0.6$ & $19.4^{\mathrm{b}} \pm 0.4$ & $25.9^{\mathrm{a}} \pm 0.4$ & $20.0^{\mathrm{b}} \pm 0.3$ & $13.3^{\mathrm{c}} \pm 0.2$ & $13.3^{\mathrm{c}} \pm 0.2$ \\
SGR (\%) & $2.9^{\mathrm{b}} \pm 0.03$ & $3.4^{\mathrm{a}} \pm 0.03$ & $2.7^{\mathrm{bc}} \pm 0.02$ & $3.2^{\mathrm{a}} \pm 0.03$ & $2.9^{\mathrm{b}} \pm 0.03$ & $2.5^{\mathrm{c}} \pm 0.02$ & $2.5^{\mathrm{c}} \pm 0.02$ \\
FCR & $1.4^{\mathrm{b}} \pm 0.02$ & $1.3^{\mathrm{a}} \pm 0.04$ & $1.6^{\mathrm{c}} \pm 0.02$ & $1.5^{\mathrm{b}} \pm 0.02$ & $1.4^{\mathrm{b}} \pm 0.03$ & $1.9^{\mathrm{d}} \pm 0.06$ & $2.2^{\mathrm{e}} \pm 0.1$ \\
PER & $2.2^{\mathrm{a}} \pm 0.02$ & $2.3^{\mathrm{a}} \pm 0.04$ & $1.8^{\mathrm{c}} \pm 0.01$ & $2.0^{\mathrm{b}} \pm 0.02$ & $2.2^{\mathrm{a}} \pm 0.01$ & $1.7^{\mathrm{c}} \pm 0.01$ & $1.4^{\mathrm{d}} \pm 0.01$ \\
ANPU (\%) & $31.6^{\mathrm{c}} \pm 0.9$ & $44.2^{\mathrm{b}} \pm 0.6$ & $25.3^{\mathrm{d}} \pm 0.3$ & $48.4^{\mathrm{a}} \pm 0.6$ & $34.2^{\mathrm{c}} \pm 0.4$ & $21.7^{\mathrm{e}} \pm 0.3$ & $15.2^{\mathrm{f}} \pm 0.2$ \\
Survival (\%) & 100 & 100 & 100 & 100 & 100 & 100 & 100 \\
\hline Digestibility & & & & & & \\
\hline Protein (\%) & $86.2^{\mathrm{a}} \pm 1.6$ & $74.4^{\mathrm{b}} \pm 1.4$ & $72.4^{\mathrm{b}} \pm 1.1$ & $73.8^{\mathrm{b}} \pm 1.2$ & $74.7^{\mathrm{b}} \pm 1.1$ & $69.1^{\mathrm{c}} \pm 1.04$ & $69.6^{\mathrm{c}} \pm 1.1$ \\
Lipid (\%) & $81.5^{\mathrm{a}} \pm 1.1$ & $73.1^{\mathrm{bc}} \pm 1.06$ & $71.3^{\mathrm{b}} \pm 1.06$ & $70.7^{\mathrm{c}} \pm 1.1$ & $74.5^{\mathrm{b}} \pm 1.1$ & $68.7 \mathrm{~d}^{\mathrm{d}} \pm 1.1$ & $68.9^{\mathrm{d}} \pm 1.1$
\end{tabular}

Data presented as Mean $\pm \mathrm{SE}(\mathrm{n}=6)$

Mean values for components with the same superscripts within the row are not significantly different $(\mathrm{p}>0.05)$

fingerlings fed with algal meal mixture (AMM) diet exhibited the lowest growth (297.2\%). However, algal meal mixture supplementation with oil $(\mathrm{AMM}+\mathrm{O})$ helped to enhance growth $(308.3 \%)$ considerably. The group fed with WS diet showed a growth rate $(400 \%)$ equivalent to that of the control diets $(402.3 \%)$. Significant increase $(\mathrm{p}<0.05)$ in growth rate was observed in the group fed with $\mathrm{AN}$ diet (565.2\%) followed by SP (500\%). Lowest FCR and highest PER and SGR were recorded with AN diet. Apparent net protein utilisation (ANPU, \%) was found to be significantly better with SP diet as compared to others.

Algal diets showed less digestibility than the control $\operatorname{diet}(\mathrm{C})$ for both protein and lipid. However, much variation was not found in the digestibility of the algal diet containing different algae (AN, NS, SP and WS). Among all the experimental diets, least protein digestibility was observed with algal meal mixture based diets (AMM+O and AMM).

No marked variation was observed between the carcass moisture content of any dietary group and initial values. Increase in the levels of carcass protein over their corresponding initial value clearly established the effect of the control and experimental diets (Table 4) on the fish. The carcass protein was found to be highest with SP (16.4\%) than AN (15.8\%) diet followed by WS, C and NS where values are equal $(14.6 \%)$. The carcass lipid content remained same compared with the initial value $(2.9 \%)$, when fish fed with C, AN, SP and WS diets. However, the carcass lipid content increased with $\mathrm{NS}, \mathrm{AMM}+\mathrm{O}$ and $\mathrm{AMM}$ over the initial value. The carcass ash content with AN, WS, AMM+O and AMM diets was found to be similar to initial value $(4.1 \%)$. Ash content increased significantly with NS, but decreased with $\mathrm{C}$ and SP diets.

The individual FA composition (expressed as $\%$ of total FA) of liver and muscle of experimental fish along with the initial FA content are shown in Table 5 and 6, respectively. Both liver and muscle fatty acids were found to be affected by dietary fatty acids. Interestingly, the change in FA followed a particular pattern both in liver and muscle as saturated fatty acid (SFA) content was found to decrease while monounsaturated (MUFA) and polyunsaturated (PUFA) content were found to increase over their respective initial values in both the tissues of all the dietary groups except the groups fed with $\mathrm{AMM}+\mathrm{O}$ and AMM. Lauric acid (12:0) and palmitic acid (16:0) were

Table 4. Carcass composition (\% wet weight basis) of tilapia fingerlings fed with experimental diet for 56 days

\begin{tabular}{lllllllll}
\hline Parameters & Initial & C & AN & NS & SP & WS & AMM $+O$ & AMM \\
\hline Moisture & $79.9^{\mathrm{a}} \pm 2.2$ & $78.5^{\mathrm{a}} \pm 1.6$ & $77.2^{\mathrm{a}} \pm 1.8$ & $76.2^{\mathrm{a}} \pm 2.0$ & $77.1^{\mathrm{a}} \pm 1.4$ & $78.2^{\mathrm{a}} \pm 1.6$ & $78.4^{\mathrm{a}} \pm 1.4$ & $78.6^{\mathrm{a}} \pm 1.9$ \\
Crude protein & $13.1^{\mathrm{c}} \pm 0.11$ & $14.7^{\mathrm{b}} \pm 0.11$ & $15.8^{\mathrm{a}} \pm 0.12$ & $14.6^{\mathrm{b}} \pm 0.11$ & $16.4^{\mathrm{a}} \pm 0.13$ & $14.7^{\mathrm{b}} \pm 0.11$ & $14.1^{\mathrm{b}} \pm 0.11$ & $13.9^{\mathrm{c}} \pm 0.11$ \\
Crude lipid & $2.9^{\mathrm{c}} \pm 0.01$ & $3.1^{\mathrm{c}} \pm 0.02$ & $2.9^{\mathrm{c} d} \pm 0.02$ & $3.3^{\mathrm{a}} \pm 0.02$ & $2.7^{\mathrm{a}} \pm 0.01$ & $3.1^{\mathrm{c}} \pm 0.03$ & $3.4^{\mathrm{a}} \pm 0.03$ & $3.2^{\mathrm{bc}} \pm 0.02$ \\
Ash & $4.1^{\mathrm{b}} \pm 0.03$ & $3.7^{\mathrm{c}} \pm 0.03$ & $4.1^{\mathrm{b}} \pm 0.04$ & $5.9^{\mathrm{a}} \pm 0.04$ & $3.8^{\mathrm{c}} \pm 0.02$ & $4.0^{\mathrm{b}} \pm 0.03$ & $4.1^{\mathrm{b}} \pm 0.02$ & $4.3^{\mathrm{b}} \pm 0.02$ \\
\hline
\end{tabular}

Data presented as Mean \pm SE $(n=6)$

Mean values for components with the same superscripts within the row are not significantly different $(p>0.05)$ 
Table 5. Fatty acid profile ( $\%$ of total fatty acid) of tilapia liver

\begin{tabular}{|c|c|c|c|c|c|c|c|c|}
\hline Fatty acid & Initial & $\mathrm{C}$ & AN & NS & SP & WS & $\mathrm{AMM}+\mathrm{O}$ & AMM \\
\hline $12: 0$ & $8.4^{\mathrm{c}} \pm 0.24$ & $6.09^{\mathrm{e}} \pm 0.16$ & $4.05^{f} \pm 0.03$ & $8.53^{\mathrm{c}} \pm 0.09$ & $7.03^{\mathrm{d}} \pm 0.2$ & $8.36^{\mathrm{c}} \pm 0.05$ & $10.19^{b} \pm 0.3$ & $12.63^{\mathrm{a}} \pm 0.3$ \\
\hline $14: 0$ & $12.9^{\mathrm{b}} \pm 0.22$ & $14.6^{\mathrm{a}} \pm 0.26$ & $10.1^{\mathrm{e}} \pm 0.06$ & $10.02^{\mathrm{e}} \pm 0.1$ & $9.55^{\mathrm{c}} \pm 0.1$ & $10.6^{\mathrm{f}} \pm 0.03$ & $14.01^{\mathrm{d}} \pm 0.1$ & $14.3^{\mathrm{a}} \pm 0.3$ \\
\hline $14: 1$ & $5.3^{\mathrm{a}} \pm 0.01$ & $4.2^{b} \pm 0.08$ & $2.01^{\mathrm{c}} \pm 0.01$ & $2.03^{\mathrm{c}} \pm 0.01$ & - & $2.08^{c} \pm 0.01$ & $4.25^{\mathrm{b}} \pm 0.01$ & $5.53^{\mathrm{a}} \pm 0.03$ \\
\hline $15: 1$ & $4.0^{\mathrm{e}} \pm 0.03$ & $3.01^{\mathrm{f}} \pm 0.02$ & $7.05^{\mathrm{b}} \pm 0.07$ & $5.64^{\mathrm{d}} \pm 0.04$ & $5.57^{\mathrm{d}} \pm 0.04$ & $9.19^{\mathrm{a}} \pm 0.1$ & $6.25^{\mathrm{c}} \pm 0.03$ & $4.09^{\mathrm{e}} \pm 0.01$ \\
\hline 16:0 & $11.8^{b} \pm 0.06$ & $12.72^{\mathrm{a}} \pm 0.6$ & $8.84^{\mathrm{de}} \pm 0.6$ & $9.31^{\mathrm{cd}} \pm 0.06$ & $9.4^{c} \pm 0.11$ & $8.14^{\mathrm{e}} \pm 0.09$ & $12.61^{\mathrm{a}} \pm 0.1$ & $12.66^{\mathrm{a}} \pm 0.2$ \\
\hline $16: 1$ & $2.0^{\mathrm{e}} \pm 0.03$ & $5.64^{\mathrm{c}} \pm 0.04$ & $7.05^{\mathrm{b}} \pm 0.07$ & $5.64^{\mathrm{c}} \pm 0.04$ & $5.57^{\mathrm{c}} \pm 0.04$ & $7.2^{\mathrm{b}} \pm 0.07$ & $4.31^{\mathrm{d}} \pm 0.02$ & $8.04^{\mathrm{a}} \pm 0.06$ \\
\hline 18:0 & $12.2^{\mathrm{a}} \pm 0.11$ & $9.63^{\mathrm{b}} \pm 0.07$ & $9.0^{\mathrm{b}} \pm 0.1$ & $9.5^{\mathrm{b}} \pm 0.16$ & $7.43^{\mathrm{c}} \pm 0.05$ & $6.51^{\mathrm{d}} \pm 0.11$ & $9.3^{\mathrm{b}} \pm 0.11$ & $9.0^{\mathrm{b}} \pm 0.1$ \\
\hline $18: \ln 7$ & $7.63^{\mathrm{a}} \pm 0.06$ & $4.03^{\mathrm{d}} \pm 0.01$ & $6.32^{b} \pm 0.04$ & $5.5^{\mathrm{c}} \pm 0.05$ & $4.92^{\mathrm{d}} \pm 0.02$ & $4.13^{\mathrm{d}} \pm 0.02$ & $5.19^{c} \pm 0.01$ & $4.18^{\mathrm{d}} \pm 0.01$ \\
\hline $18: \ln 9$ & $8.49^{\mathrm{a}} \pm 0.06$ & $7.08^{b} \pm 0.06$ & $4.7^{\mathrm{e}} \pm 0.02$ & $6.53^{\mathrm{c}} \pm 0.06$ & $3.63^{\mathrm{f}} \pm 0.01$ & $4.43^{\mathrm{e}} \pm 0.11$ & $4.1^{\mathrm{ef}} \pm 0.01$ & $5.1^{\mathrm{d}} \pm 0.01$ \\
\hline $18: 2 \mathrm{n} 6$ & $3.79^{c} \pm 0.02$ & $6.0^{\mathrm{a}} \pm 0.02$ & $6.4^{\mathrm{a}} \pm 0.05$ & $3.18^{\mathrm{c}} \pm 0.03$ & $3.19^{c} \pm 0.03$ & $4.17^{b} \pm 0.03$ & $2.61^{\mathrm{d}} \pm 0.1$ & $3.18^{\mathrm{e}} \pm 0.01$ \\
\hline $18: 3 n 6$ & - & - & - & - & $6.31^{\mathrm{a}} \pm 0.06$ & - & $2.01^{\mathrm{c}} \pm 0.01$ & $2.62^{b} \pm 0.01$ \\
\hline $18: 3 n 3$ & $3.78^{\mathrm{cd}} \pm 0.02$ & $4.13^{b} \pm 0.03$ & $5.88^{\mathrm{a}} \pm 0.05$ & $3.1^{\mathrm{d}} \pm 0.02$ & $4.02^{\mathrm{b}} \pm 0.02$ & $5.31^{\mathrm{a}} \pm 0.04$ & $2.16^{\mathrm{e}} \pm 0.01$ & $2.24^{\mathrm{e}} \pm 0.01$ \\
\hline 20:0 & $2.26^{\mathrm{f}} \pm 0.01$ & $5.54^{\mathrm{d}} \pm 0.02$ & $8.84^{b} \pm 0.06$ & $6.64^{\mathrm{c}} \pm 0.01$ & $6.14^{\mathrm{cd}} \pm 0.04$ & $4.39^{\mathrm{e}} \pm 0.08$ & $9.57^{\mathrm{a}} \pm 0.1$ & $1.18^{\mathrm{g}} \pm 0.01$ \\
\hline $20: 2$ n6 & $3.44^{\mathrm{b}} \pm 0.01$ & $3.16^{\mathrm{b}} \pm 0.02$ & $2.06^{\mathrm{c}} \pm 0.01$ & $3.34^{b} \pm 0.02$ & $2.26^{\mathrm{c}} \pm 0.02$ & $5.3^{\mathrm{a}} \pm 0.04$ & $2.04^{c} \pm 0.07$ & $2.14^{c} \pm 0.02$ \\
\hline $20: 3$ n6 & $3.39^{\mathrm{b}} \pm 0.02$ & $3.4^{\mathrm{b}} \pm 0.01$ & $4.8^{\mathrm{a}} \pm 0.03$ & $4.01^{\mathrm{a}} \pm 0.03$ & $4.06^{\mathrm{a}} \pm 0.01$ & $3.04^{b} \pm 0.02$ & $1.96^{\mathrm{c}} \pm 0.02$ & $1.88^{c} \pm 0.01$ \\
\hline $20: 3 n 3$ & $1.7^{\mathrm{bc}} \pm 0.03$ & $1.26^{\mathrm{c}} \pm 0.05$ & $2.2^{\mathrm{a}} \pm 0.09$ & $2.01^{\mathrm{ab}} \pm 0.03$ & $1.37^{\mathrm{c}} \pm 0.03$ & $2.9^{\mathrm{a}} \pm 0.05$ & $1.04^{\mathrm{d}} \pm 0.03$ & $0.81^{\mathrm{e}}$ \\
\hline $20: 4 n 6$ & $3.18^{\mathrm{b}} \pm 0.02$ & $4.16^{\mathrm{a}} \pm 0.04$ & $3.48^{\mathrm{b}} \pm 0.03$ & $4.39^{\mathrm{a}} \pm 0.04$ & $4.15^{\mathrm{a}} \pm 0.02$ & $2.19^{c} \pm 0.02$ & $2.51^{\mathrm{c}} \pm 0.02$ & $1.64^{\mathrm{d}} \pm 0.02$ \\
\hline $20: 5 n 3$ & $1.69^{\mathrm{a}} \pm 0.01$ & $1.08^{\mathrm{bc}} \pm 0.01$ & $1.65^{\mathrm{a}} \pm 0.01$ & $0.9^{c} \pm 0.01$ & $1.01^{\mathrm{bc}} \pm 0.01$ & $1.42^{\mathrm{ab}} \pm 0.01$ & $1.01^{\mathrm{bc}} \pm 0.01$ & $0.96^{\mathrm{c}} \pm 0.01$ \\
\hline $22: 6 n 3$ & $0.3^{\mathrm{c}}$ & $0.39^{\mathrm{b}}$ & $0.38^{\mathrm{b}}$ & $0.21^{\mathrm{d}}$ & $0.16^{\mathrm{e}}$ & $0.64^{\mathrm{a}}$ & - & - \\
\hline $24: \ln 9$ & - & $1.06^{\mathrm{d}} \pm 0.01$ & $1.61^{\mathrm{d}} \pm 0.04$ & $6.83^{\mathrm{b}} \pm 0.05$ & $9.25^{\mathrm{a}} \pm 0.1$ & $6.12^{b} \pm 0.04$ & $2.86^{\mathrm{c}} \pm 0.01$ & $2.95^{\mathrm{c}} \pm 0.02$ \\
\hline$\Sigma \mathrm{FA}$ & $96.25 \pm 2.4$ & $97.15 \pm 2.5$ & $96.4 \pm 3.1$ & $97.3 \pm 2.11$ & $95.02 \pm 2.5$ & $96.11 \pm 3.01$ & $97.98 \pm 2.2$ & $95.14 \pm 2.1$ \\
\hline Others & $3.71 \pm 0.04$ & $2.85 \pm 0.03$ & $3.6 \pm 0.01$ & $2.7 \pm 0.1$ & $4.98 \pm 0.04$ & $3.89 \pm 0.02$ & $2.02 \pm 0.02$ & $4.86 \pm 0.02$ \\
\hline$\Sigma$ SFA & $47.56^{b} \pm 0.6$ & $48.57^{b} \pm 0.5$ & $40.84^{\mathrm{cd}} \pm 0.4$ & $43.99^{c} \pm 0.4$ & $39.55^{\mathrm{d}} \pm 0.4$ & $38.01^{b c} \pm 0.3$ & $55.7^{a} \pm 0.6$ & $49.78^{b} \pm 0.6$ \\
\hline$\Sigma$ MUFA & $27.42^{\mathrm{bc}} \pm 0.2$ & $25.0^{\mathrm{b}} \pm 0.2$ & $28.74^{\mathrm{bc}} \pm 0.3$ & $32.17^{\mathrm{a}} \pm 0.2$ & $28.94^{\mathrm{bc}} \pm 0.2$ & $33.14^{a} \pm 0.3$ & $27.0^{\mathrm{bc}} \pm 0.3$ & $29.9^{b} \pm 0.24$ \\
\hline$\Sigma$ PUFA & $21.27^{\mathrm{c}} \pm 0.2$ & $23.58^{\mathrm{ab}} \pm 0.2$ & $26.85^{\mathrm{a}} \pm 0.3$ & $21.14^{\mathrm{b}} \pm 0.3$ & $26.53^{\mathrm{a}} \pm 0.3$ & $25.0^{\mathrm{a}} \pm 0.3$ & $15.34^{\mathrm{c}} \pm 0.2$ & $15.47^{\mathrm{c}} \pm 0.1$ \\
\hline$\Sigma \mathrm{n} 3$ & $7.47^{\mathrm{b}} \pm 0.08$ & $6.86^{\mathrm{c}} \pm 0.06$ & $10.1^{\mathrm{a}} \pm 0.2$ & $6.22^{\mathrm{c}} \pm 0.07$ & $6.56^{\mathrm{c}} \pm 0.04$ & $10.27^{\mathrm{a}} \pm 0.1$ & $4.21^{\mathrm{d}} \pm 0.03$ & $4.01^{\mathrm{d}} \pm 0.02$ \\
\hline$\Sigma \mathrm{n} 6$ & $13.8^{\mathrm{c}} \pm 0.1$ & $16.72^{\mathrm{b}} \pm 0.1$ & $16.75^{\mathrm{b}} \pm 0.2$ & $14.92^{\mathrm{c}} \pm 0.2$ & $19.97^{\mathrm{a}} \pm 0.2$ & $14.7^{\mathrm{c}} \pm 0.1$ & $11.13^{\mathrm{d}} \pm 0.1$ & $11.46^{\mathrm{d}} \pm 0.1$ \\
\hline $\mathrm{n} 3 / \mathrm{n} 6$ & $0.54^{\mathrm{c}}$ & $0.41^{\mathrm{d}}$ & $0.60^{\mathrm{b}}$ & $0.41^{\mathrm{d}}$ & $0.33^{\mathrm{e}}$ & $0.7^{\mathrm{a}}$ & $0.38^{\mathrm{e}}$ & $0.35^{\mathrm{e}}$ \\
\hline
\end{tabular}

Data presented as Mean \pm SE $(n=6)$. Mean values for components with the same superscripts within the row are not significantly different ( $\mathrm{p}>0.05)$

predominant among SFAs in muscle and liver, respectively. Among MUFAs, pentadecanoic acid (15:1) and palmitoloic acid (16:1) were predominant in liver while in muscle, oleic acid (18:1 n9) and nervonic acid (24:1n9) were predominant.

In general, the level of n- 6 was found to be higher than n-3 in both muscle and liver in all dietary groups. In tissues of the groups fed with SP and AMM+O, n- 6 FA was double in quantity than n-3 FA. Among the n-6 FAs, gamma linolenic acid (18:3n6) accumulated in both muscle and liver of the groups fed with SP either individually or in mixture (AMM and $\mathrm{AMM}+\mathrm{O}$ ). No such uniform trend was noticed in the EPA and DHA level in any dietary groups for both the tissues. However, WS could induce higher EPA and DHA content in both liver and muscle among all diets.

\section{Discussion}

Nutritive value of freshwater algal meal as a nutrient source in pelleted feed was demonstrated in this study. Preparation of algal meal described in the study was reported earlier (Mishra, 2000) without affecting the iodine value of the lipids. It was possible to include algal meal up to $40 \%$ of the total diet without any adverse effect on the growth of tilapia which is in agreement with earlier report on rohu (Mishra, 2000). However, Mustafa et al. (1995) included algal meal up to a level of 5\% for red sea bream. Wee and Wang (1987) successfully used soaked Leucaena leaf meal up to $25 \%$ of the total protein which accounts up to a level of $31 \%$ of the total diet in the tilapia diet. Based on the nutritional value of marine algae (Brown et al., 1997), their use in mariculture has been emphasised (Mustafa et al., 1995). However, the use of freshwater algae for the culture of fish is limited which may be because of the fact that their nutritional value is not well known (Ahlgren, 1992). It has been reported previously that the average final weight of Nile tilapia fed by zooplankton and artificial feed in monoculture was 145 and $110 \mathrm{~g}$, respectively (Abd EI and Hassan, 2011). In the present study, highest growth of 565.2 and $500 \%$ was observed by feeding algae like AN and SP. Other two algae such as NS and WS increased growth rate up to $400 \%$ which is similar to rice bran, GNOC and soyabean meal based control diet. Sumi (2011) found a survival rate of $94 \%$ by feeding $35 \%$ protein 
Table 6. Fatty acid profile (\% of total fatty acid) of tilapia muscle

\begin{tabular}{|c|c|c|c|c|c|c|c|c|}
\hline Fatty acid & Initial & $\mathrm{C}$ & AN & NS & SP & WS & $\mathrm{AMM}+\mathrm{O}$ & AMM \\
\hline $12: 0$ & $12.74^{\mathrm{ab}} \pm 0.2$ & $11.48^{\mathrm{b}} \pm 0.1$ & $10.8^{c} \pm 0.1$ & $10.19^{c} \pm 0.2$ & $9.42^{\mathrm{d}} \pm 0.02$ & $9.41^{\mathrm{d}} \pm 0.11$ & $12.63^{\mathrm{ab}} \pm 0.1$ & $13.27^{\mathrm{a}} \pm 0.2$ \\
\hline $14: 0$ & $6.99^{\mathrm{d}} \pm 0.05$ & $2.14^{\mathrm{g}} \pm 0.02$ & $4.01^{\mathrm{f}} \pm 0.06$ & $6.32^{\mathrm{de}} \pm 0.1$ & $6.15^{\mathrm{e}} \pm 0.06$ & $7.85^{\mathrm{c}} \pm 0.1$ & $9.03^{\mathrm{b}} \pm 0.2$ & $12.36^{\mathrm{a}} \pm 0.2$ \\
\hline $14: 1$ & $4.11^{\mathrm{c}} \pm 0.01$ & $2.11^{\mathrm{e}} \pm 0.01$ & $3.9^{\mathrm{d}} \pm 0.01$ & $4.38^{\mathrm{c}} \pm 0.02$ & $6.68^{b} \pm 0.02$ & $4.26^{\mathrm{c}} \pm 0.04$ & $6.14^{b} \pm 0.02$ & $7.22^{\mathrm{a}} \pm 0.02$ \\
\hline $15: 1$ & $5.08^{\mathrm{d}} \pm 0.05$ & $4.37^{\mathrm{e}} \pm 0.04$ & $4.49^{\mathrm{e}} \pm 0.07$ & $5.53^{\mathrm{d}} \pm 0.15$ & $9.53^{b} \pm 0.06$ & $8.09^{\mathrm{c}} \pm 0.06$ & $9.01^{b} \pm 0.05$ & $10.19^{\mathrm{a}} \pm 0.1$ \\
\hline $16: 0$ & $10.92^{\mathrm{ab}} \pm 0.1$ & $9.44^{\mathrm{c}} \pm 0.08$ & $8.56^{\mathrm{d}} \pm 0.08$ & $9.54^{\mathrm{c}} \pm 0.09$ & $10.0^{\mathrm{bc}} \pm 0.1$ & $9.47^{\mathrm{c}} \pm 0.1$ & $11.18^{\mathrm{a}} \pm 0.1$ & $9.2^{\mathrm{c}} \pm 0.1$ \\
\hline $16: 1$ & $8.06^{b} \pm 0.05$ & $6.12^{\mathrm{c}} \pm 0.01$ & $6.43^{c} \pm 0.07$ & $10.88^{\mathrm{a}} \pm 0.2$ & $8.88^{b} \pm 0.06$ & $6.31^{\mathrm{c}} \pm 0.03$ & $6.18^{\mathrm{c}} \pm 0.08$ & $4.65^{\mathrm{d}} \pm 0.01$ \\
\hline $17: 1$ & $4.11^{b} \pm 0.04$ & $2.08^{\mathrm{d}} \pm 0.02$ & $1.9^{\mathrm{e}} \pm 0.01$ & $2.38^{\mathrm{d}} \pm 0.02$ & $8.68^{\mathrm{a}} \pm 0.02$ & $1.2^{\mathrm{f}} \pm 0.01$ & $4.01^{\mathrm{b}} \pm 0.05$ & $3.6^{c} \pm 0.04$ \\
\hline 18:0 & $8.02^{b} \pm 0.2$ & $8.27^{b} \pm 0.06$ & $6.35^{\mathrm{c}} \pm 0.06$ & $8.33^{b} \pm 0.09$ & $4.32^{\mathrm{d}} \pm 0.03$ & $6.07^{c} \pm 0.06$ & $8.25^{b} \pm 0.1$ & $10.04^{\mathrm{a}} \pm 0.2$ \\
\hline $18: \ln 7$ & $6.01^{\mathrm{c}} \pm 0.03$ & $7.56^{\mathrm{b}} \pm 0.03$ & $8.98^{\mathrm{a}} \pm 0.07$ & $6.54^{\mathrm{c}} \pm 0.03$ & $4.06^{\mathrm{e}} \pm 0.02$ & $5.8^{\mathrm{d}} \pm 0.04$ & $6.92^{c} \pm 0.09$ & $7.63^{b} \pm 0.04$ \\
\hline $18: \ln 9$ & $6.02^{\mathrm{d}} \pm 0.14$ & $9.2^{\mathrm{a}} \pm 0.09$ & $8.18^{b} \pm 0.09$ & $6.4^{\mathrm{d}} \pm 0.06$ & $7.41^{\mathrm{c}} \pm 0.01$ & $8.92^{\mathrm{b}} \pm 0.04$ & $8.18^{\mathrm{b}} \pm 0.08$ & $3.36^{\mathrm{e}} \pm 0.02$ \\
\hline $18: 2 \mathrm{n} 6$ & $3.86^{\mathrm{c}} \pm 0.06$ & $3.39^{\mathrm{c}} \pm 0.02$ & $4.33^{\mathrm{ab}} \pm 0.03$ & $4.24^{\mathrm{b}} \pm 0.06$ & $4.89^{\mathrm{a}} \pm 0.06$ & $3.41^{\mathrm{c}} \pm 0.04$ & $1.19^{\mathrm{d}} \pm 0.01$ & $1.43^{\mathrm{d}} \pm 0.01$ \\
\hline $18: 3 n 6$ & - & - & - & - & $3.48^{\mathrm{a}} \pm 0.09$ & - & $1.40^{\mathrm{b}} \pm 0.01$ & $1.58^{\mathrm{b}} \pm 0.01$ \\
\hline $18: 3 n 3$ & $3.62^{\mathrm{c}} \pm 0.07$ & $4.18^{\mathrm{b}} \pm 0.07$ & $4.02^{b} \pm 0.09$ & $3.19^{c} \pm 0.04$ & $3.07^{\mathrm{c}} \pm 0.06$ & $4.81^{\mathrm{a}} \pm 0.05$ & $2.18^{\mathrm{d}} \pm 0.02$ & $2.18^{\mathrm{d}} \pm 0.01$ \\
\hline 20:0 & $4.83^{\mathrm{c}} \pm 0.04$ & $6.0^{\mathrm{a}} \pm 0.07$ & $4.58^{\mathrm{cd}} \pm 0.14$ & $4.16^{\mathrm{d}} \pm 0.01$ & $3.18^{\mathrm{e}} \pm 0.05$ & $4.13^{\mathrm{d}} \pm 0.02$ & $5.39^{b} \pm 0.01$ & $6.59^{\mathrm{a}} \pm 0.05$ \\
\hline $20: 2 \mathrm{n} 6$ & $3.1^{\mathrm{c}} \pm 0.04$ & $4.83^{\mathrm{a}} \pm 0.05$ & $4.9^{\mathrm{a}} \pm 0.08$ & $3.62^{b} \pm 0.05$ & $4.36^{\mathrm{a}} \pm 0.04$ & $4.81^{\mathrm{a}} \pm 0.05$ & $2.18^{\mathrm{d}} \pm 0.02$ & $1.08^{\mathrm{e}} \pm 0.01$ \\
\hline $20: 3 n 6$ & $2.19^{\mathrm{d}} \pm 0.02$ & $2.89^{b} \pm 0.01$ & $3.54^{\mathrm{a}} \pm 0.04$ & $2.44^{\mathrm{cd}} \pm 0.01$ & $2.86^{\mathrm{b}} \pm 0.03$ & $2.67^{\mathrm{bc}} \pm 0.03$ & $1.88^{\mathrm{e}} \pm 0.01$ & $1.17^{\mathrm{f}} \pm 0.02$ \\
\hline $20: 3 n 3$ & $2.06^{b c} \pm 0.08$ & $3.93^{b} \pm 0.08$ & $4.04^{\mathrm{a}} \pm 0.1$ & $2.03^{\mathrm{d}} \pm 0.07$ & $1.34^{\mathrm{c}} \pm 0.05$ & $2.67^{b} \pm 0.1$ & $1.14^{\mathrm{cd}} \pm 0.02$ & $1.14^{\mathrm{d}} \pm 0.01$ \\
\hline $20: 4 n 6$ & $3.41^{\mathrm{b}} \pm 0.04$ & $2.63^{\mathrm{c}} \pm 0.01$ & $3.04^{b} \pm 0.02$ & $2.37^{\mathrm{c}} \pm 0.01$ & $2.69^{c} \pm 0.06$ & $3.92^{\mathrm{a}} \pm 0.06$ & $2.82^{\mathrm{c}} \pm 0.04$ & $1.76^{\mathrm{d}} \pm 0.02$ \\
\hline $20: 5 \mathrm{n} 3$ & $1.52^{\mathrm{b}} \pm 0.02$ & $1.19^{\mathrm{c}} \pm 0.01$ & $1.01^{\mathrm{d}} \pm 0.02$ & $1.05^{\mathrm{d}} \pm 0.02$ & $1.06^{\mathrm{d}} \pm 0.1$ & $1.63^{\mathrm{a}} \pm 0.02$ & $0.9^{\mathrm{e}} \pm 0.01$ & $0.7^{\mathrm{e}}$ \\
\hline $22: 6 n 3$ & $0.3^{\mathrm{c}}$ & $0.5^{\mathrm{a}}$ & $0.42^{\mathrm{b}}$ & $0.2^{\mathrm{d}}$ & $0.2^{\mathrm{d}}$ & $0.5^{\mathrm{a}}$ & - & - \\
\hline $24: \ln 9$ & $3.69^{\mathrm{c}} \pm 0.04$ & $6.98^{\mathrm{a}} \pm 0.08$ & $4.03^{\mathrm{b}} \pm 0.01$ & $3.39^{\mathrm{c}} \pm 0.05$ & $4.15^{\mathrm{b}} \pm 0.03$ & $3.43^{c} \pm 0.04$ & $1.83^{\mathrm{d}} \pm 0.01$ & $1.51^{\mathrm{d}} \pm 0.01$ \\
\hline$\Sigma$ FA & $96.53 \pm 3.1$ & $97.21 \pm 3.8$ & $95.61 \pm 3.3$ & $94.28 \pm 2.9$ & $97.73 \pm 4.2$ & $98.16 \pm 2.6$ & $98.43 \pm 3.34$ & $97.06 \pm 3.18$ \\
\hline Others & $3.47 \pm 0.06$ & $2.79 \pm 0.04$ & $4.39 \pm 0.04$ & $5.2 \pm 0.1$ & $2.27 \pm 0.02$ & $1.84 \pm 0.07$ & $1.57 \pm 0.04$ & $2.94 \pm 0.08$ \\
\hline$\Sigma$ SFA & $43.5^{\mathrm{b}} \pm 0.6$ & $37.33^{\mathrm{cd}} \pm 0.5$ & $34.3^{\text {cd }} \pm 0.4$ & $38.54^{\mathrm{c}} \pm 0.5$ & $33.07^{\mathrm{d}} \pm 0.4$ & $36.93^{\mathrm{cd}} \pm 0.4$ & $46.48^{b} \pm 0.6$ & $51.46^{\mathrm{a}} \pm 0.6$ \\
\hline$\Sigma$ MUFA & $32.97^{\mathrm{c}} \pm 0.2$ & $36.34^{b} \pm 0.3$ & $36.01^{\mathrm{b}} \pm 0.3$ & $37.12^{\mathrm{ab}} \pm 0.3$ & $40.71^{\mathrm{a}} \pm 0.2$ & $36.81^{b} \pm 0.2$ & $38.26^{\mathrm{a}} \pm 0.3$ & $34.56^{\mathrm{bc}} \pm 0.4$ \\
\hline$\Sigma$ PUFA & $20.12^{b} \pm 0.3$ & $23.54^{\mathrm{a}} \pm 0.3$ & $25.30^{\mathrm{a}} \pm 0.6$ & $19.1^{\mathrm{b}} \pm 0.24$ & $23.95^{\mathrm{a}} \pm 0.4$ & $24.42^{\mathrm{a}} \pm 0.4$ & $13.69^{c} \pm 0.2$ & $11.04 \mathrm{~d} \pm 0.1$ \\
\hline$\Sigma \mathrm{n} 3$ & $7.56^{\mathrm{b}} \pm 0.03$ & $9.8^{\mathrm{a}} \pm 0.03$ & $9.49^{\mathrm{a}} \pm 0.06$ & $6.47^{\mathrm{c}} \pm 0.02$ & $5.67^{\mathrm{d}} \pm 0.04$ & $9.61^{\mathrm{a}} \pm 0.06$ & $4.22^{\mathrm{e}} \pm 0.02$ & $4.02^{\mathrm{e}} \pm 0.04$ \\
\hline$\sum \mathrm{n} 6$ & $12.56^{\mathrm{cd}} \pm 0.1$ & $13.74^{\mathrm{bc}} \pm 0.1$ & $15.81^{\mathrm{b}} \pm 0.2$ & $12.63^{\mathrm{cd}} \pm 0.1$ & $18.28^{\mathrm{a}} \pm 0.3$ & $14.81^{\mathrm{bc}} \pm 0.2$ & $9.47^{\mathrm{de}} \pm 0.1$ & $7.02^{\mathrm{e}} \pm 0.08$ \\
\hline $\mathrm{n} 3 / \mathrm{n} 6$ & $0.60^{c}$ & $0.71^{\mathrm{b}}$ & $0.60^{c}$ & $0.51^{\mathrm{d}}$ & $0.316^{\mathrm{f}}$ & $0.85^{\mathrm{a}}$ & $0.45^{\mathrm{e}}$ & $0.57^{\mathrm{d}}$ \\
\hline
\end{tabular}

Data presented as Mean $\pm \mathrm{SE}(\mathrm{n}=6)$.

Mean values for components with the same superscripts within the row are not significantly different $(p>0.05)$

diet based on fish meal. In the present study, $32 \%$ protein diet was used. Weight gain, SGR (\%), ANPU (\%) and survival $(\%)$ of tilapia were significantly $(\mathrm{p}<0.05)$ higher and better feed utilisation with lower FCR value was observed in fish fed with meat and bone meal which was used as sole source of protein base (Siddika et.al., 2012). Schroeder et al. (1990) reported that natural food contributed between 300 and $500 \mathrm{~g} \mathrm{~kg}^{-1}$ of growth when tilapia was supplemented with artificial feeds in fertilised ponds.

The low capability of tilapia to digest algal meal based diets observed in the present study is comparable to that reported for Leucaena leaf meal based diets (Wee and Wang, 1987) and diets with plant ingredients (Keembiyehetti and De Silva, 1993) for tilapia or algal meal based diets for rohu (Mishra, 2000). Even though protein digestibility was less compared to control diet, growth was better in the present study with AN and SP diet which may be due to better protein assimilation and utilisation. In the present study, the digestibility further reduced when the diet was completely based on algal meal mixture. However, higher digestive coefficients were reported by Kirilenko et al. (1975) in O. mossambicus for the crude protein and natural fat of some microalgae (Microcystis aeruginosa $99 \%$ and Aphanizomeron flosaque 1\% and natural foods (Chlorella and Daphnia). As the culture methods for Microcystis, Chlorella and Daphnia could not be standardised, these could not be used in the present study. Significantly higher digestibility values (70-82\%) have been recorded for cyanobacteria than chlorophyceae (37.52\%) in tilapia (Dempster et al., 1993).

Noticeable differences were observed in the growth rate of tilapia fed different algal meal based diets in the present study. The growth performance and feed utilisation efficiencies of fish fed with AN diet was found to be best among all the diet. Lower growth in WS could be attributed to poor protein digestibility. Buddington (1979) observed that cellulose and chitin of plant fibres were poorly digested 
by three species of tilapia viz., O. aureus, O. mossambicus and $O$. niloticus. Herbivorous and omnivorous fish generally have a low digestion efficiency when fed natural diets as compared to carnivorous fish (Buddington, 1979). Obviously, prepared diets in the present report facilitated digestion in tilapia. Only $\mathrm{AMM}+\mathrm{O}$ or $\mathrm{AMM}$ could not induce much growth. Under similar experimental conditions and diets, WS diet performed best in rohu (Mishra, 2000) but in tilapia, AN diet was found to be the best. However, in both cases SP diet showed moderate impact. It is interesting to note that tilapia can maintain growth rate consuming only algal meal mixture (AMM) diet.

The growth and feed utilisation efficiencies in tilapia fed AMM diet did not improve much by addition of fat sources, which may be due to protein sparing effect of lipid. Addition of supplemental protein to AMM without supplementation of fat sources improved growth and feed utilisation in rohu (Mishra, 2000). Reduced feed efficiencies with increase in percentage of leaf meal in tilapia diet was reported earlier (Wee and Wang, 1987). O. aureus, and O. niloticus showed poor FCR with dehydrated alfalfa and salt bush (Atriplex) leaves (Yousif et al., 1994) and plant ingredients (Keembiyehetty and De Silva, 1993), respectively. On the other hand, Zohar et al. (1985) recorded better conversion efficiencies for tilapia under intensive culture system with plant ingredient based diets. Such variation in the feed efficiencies may be possibly caused by poor nutrient availability and fibre content. Thus proper care should be taken to avoid plant materials with high fibre content and to process the ingredient for better digestion by the fish.

Except moisture, carcass composition was found to be affected by the various algal meal based diets. Significant increase was observed in carcass protein in the present study due to SP and AN diets. The WS and NS as well as control diets induced similar rate of protein to carcass conversion. Diet AMM+O deposited higher carcass lipid while SP diet led to least lipid levels in carcass. This showed that SP diet is better to produce tilapia with high protein and less lipid. But ash content did not follow any trend. It was recognised earlier that the effects of the diets had been masked by an unquantified input of food energy from the natural production of micro algae within the tilapia culture tank (Hanley, 1991). In this study, lipid content of the diet was almost maintained at same level, still then both protein and lipid of carcass was affected by the diet, which may be because of other nutrients in the diet.

Warm water fish have an n-3 fatty acid requirement but they are less sensitive to deficiency than cold-water species (Satoh et al., 1987). Tilapia zilli, requires approximately 1\% n-6 fatty acids in the diet (Kanazawa et al., 1980) and the requirement can be met with either $18: 2 \mathrm{n} 6$ or $20: 4 \mathrm{n} 6$. Similar results were observed in $O$. niloticus (Takeuchi et al., 1983). Though $T$. aureus requires relatively high levels of $n-6$ fatty acids, the requirement could be reduced when n-3 fatty acids are present (Stickney and Mc Geachin, 1983). Even though the dietary requirements of n-3 and n-6 FAs were not studied in the present investigation, proper attention has been paid to formulate the diet to provide adequate quantities of the essential FAs to meet the requirements of this fish and considering the digestive capability of the fish to digest the plant materials.

The results of this investigation depict the positive effect of dietary FAs with respect to MUFA and PUFA on the tissue and negative effects of SFA. This states that the dietary nutrients were used by the candidate species as was observed earlier for rohu (Mishra, 2000). Significant relative decrease of SFA (14:0) in both liver and muscle of red tilapia (De Silva et al., 1997) and catfish (Zamal and Ollevier, 1994) during starvation has been reported earlier. This study observed similar effects on SFA level in fed tilapia. Even though lot of work has been done on the effect of starvation on the FA content of tilapia, reports are limited on the dietary effect on tissue FA profile (De Silva et al., 1997). In fed catfish, liver SFA (14:0) decreased significantly compared with control, whereas MUFA (16:1n9 and 18:1n9) and PUFA (20:5n3 and $22: 6 \mathrm{n} 3)$ remained same up to the end of the feeding study and in muscle, the SFA and MUFA remained similar throughout the experiment with no significant variation of PUFA over the initial level (Zamal and Ollevier, 1994). The total FA in liver and muscle of hybrid tilapia fed with commercial pellets, were reported to decrease and increase, respectively with growth, while significant proportional increase of individual SFA and MUFA in liver was noticed up to $24^{\text {th }}$ day of feeding (De Silva et al., 1997). In the present study on tilapia, significant changes were observed in SFA and MUFA in both liver and muscle.

Accumulation of gamma linolenic acid (18:3n6) in both muscle and liver of tilapia fed with Spirulina either individually (SP diet) or in algal meal mixture emphasised the utilisation of dietary fatty acid by tilapia as was observed for rohu with algal diets (Mishra, 2000). Even though AN fed group showed slightly higher content of PUFA (20:5n3 and 22:6n3), no significant changes in the PUFA was observed in other dietary groups over that of the initial value in tilapia which was in contrast to the observation on rohu fed with algal diets (Mishra, 2000). In general, the level of n6 FA was found more than $\mathrm{n} 3 \mathrm{FA}$ in both the tissues of tilapia in all the dietary groups. The accumulation of more n6 FA by tilapia may be because of the physiological adoption of this species to the herbivorous feeding habit. Further, onfarm trials using the algal diets are necessary prior to use of these feeds for commercial culture of tilapia. 


\section{Acknowledgements}

The authors gratefully acknowledge the support of the research team of Algal Research Laboratory, Berhampur University, Odisha for providing the pure strains of algae for this research work.

\section{References}

Abd El, A. and Hassan, R. 2011. Zooplankton as natural live food for three different fish species under concrete ponds with monoand polyculture conditions. Egypt. J. Aquac., 1(1): 27-40.

Ahlgren, G. 1992. Fatty acid content and chemical composition of freshwater microalgae. J. Phycol., 28: 37-50.

Appler, H. N. 1985. Evaluation of Hydrodictyon reticulatum as protein source in feed for Tilapia Oreochromis niloticus and Tilapia zilli. J. Fish. Biol., 27: 327-334.

AOAC 1975. Official methods of analysis, $12^{\text {th }}$ edn. Association of Official Analytical Chemists Washington D. C., 1094 pp.

Boberg, M., Caroon, L. B. and Vessby, B. 1985. Platelet fatty acid composition in plasma and to serum lipoprotein lipids in healthy subjects with special reference to the linolenic acid pathways. Clin. Sci., 68: 581-587.

Brown, M. R., Jeffery, S. W., Volkman, J. K. and Dunstan, G. A. 1997. Nutritional properties of microalgae for mariculture. Aquaculture, 151: 315-331.

Buddington, R. K. 1979. Digestion of an aquatic macrophyte by Tilapia zilli (Gervais). J. Fish. Biol., 15: 449-455.

De Silva, S. S., Gunasekera, R. M. and Austin, C. M. 1997. Changes in fatty acid profile of hybrid red tilapia, Oreochromis mossambicus x O. niloticus, subjected to short-term starvation and a comparison with changes in seawater raised fish. Aquaculture, 153: 273-290.

Dempster, P. W., Beveridge, M. C. M. and Baird, D. J. 1993. Herbivory in the tilapia Oreochromis niloticus (L): A comparison of feeding rates on periphyton and phytoplankton (Microcystis aeruginosa). J. Fish Biol., 43: 385-392.

Devadasan, K. 2002. Proteins and lipid of muscle products and their changes during processing and preservation. In: Gopakumar, K. (Ed.), Textbook of fish processing and technology. Indian Council of Agricultural Research, New Delhi. 491 pp.

Duncan, D. B. 1955. Multiple range and multiple F-test. Biometrics, 11: $1-42$.

FAO 2014. The state of world fisheries and aquaculture. Food and Agriculture Organisation of the United Nations, Rome, Italy, $223 \mathrm{pp}$.

Furukawa, A., and Tsukahara, H. 1966. On the acid digestion method of determination of chromic oxide as an indicator substance in the study of digestibility of fish. Bull. Jpn. Soc. Sci. Fish., 32: 502-506.
Gopakumar, K. and Nair, R. M. 1972. Fatty acid composition of eight species of Indian marine fish. J. Sci. Food Agric., 23: 493-496.

Hanley, F. 1991. Effect of feeding supplementary diets containing varying levels of lipid on growth, food conversion and body composition of nile tilapia Oreochromis niloticus (L). Aquaculture, 93: 323-334.

Hepher, B., Sandbank, E. and Shelef, G. 1978. Alternative protein sources for warm water fish diets. In: EIFAC Symposium on Finfish Nutrition and Feed Technology, June 1975, Hamburg., EIFAC/78/ Symp. R. 11.2.

Kanazawa, A., Teshima, S., Sakamoto, M. and Awal, M. A. 1980. Requirements of Tilapia zillii for essential fatty acids. Bull. Jpn. Soc. Sci. Fish., 46: 1353-1356.

Keembiyehetty, C. N. and De Silva, S. S. 1993. Performance of juvenile Oreochromis niloticus (L) reared on diets containing cowpea (Vigna catiang) and blackgram (Phaseolus mungo) seeds. Aquaculture, 112: 207-215.

Kirilenko, N. S., Mel'nikov, G. B., Grinberg, L. R. and Nazimirova, N. I. 1975. The digestibility of the crude protein and neutral fat of some microalgae by Tilapia mossambica. J. Ichthyol., 15: 151-155.

Lund, I., Hoglund, E., Ebbesson, L. O. E. and Skov, P. V. 2014. Dietary LC-PUFA deficiency early in ontogeny induces behavioural changes in Pike perch (Sander lucioperca) larva and fry. Aquaculture, 432: 453-461.

Matty, A. J. and Smith, P. 1978. Evaluation of a yeast, a bacterium and an alga as a protein source for rainbow trout I. Effect of protein level on growth, gross conversion efficiency and protein conversion efficiency. Aquaculture, 14: 235-246.

Meyer, E. and Walther, A. 1988. Methods for the estimation of protein lipid, carbohydrate and chitin levels in freshwater invertebrates. Arch. Hydrobiol., 113: 161-167.

Mishra, K. 2000. Effect of dietary lipid and lipid-temperature interaction on the growth performance, percentage lipid, fatty acid composition of Indian major carp Labeo rohita (Hamilton) using algal meal additive diets. Ph. D. Thesis, Berhampur University, India, 258 pp.

Mishra, K. and Samantaray, K. 2000. Fatty acid composition of six freshwater microalgae. J. Aquac., 8: 9-16.

Mishra, K. and Samantaray, K. 2004. Interacting effect of dietary lipid level and temperature on growth, body composition and fatty acid profile of rohu Labeo rohita (Ham.). Aquac. Nutr., 10: 359-369.

Mustafa, G. Md., Wakamatsu, S., Takeda, T., Umino, T. and Nakagawa, H. 1995. Effects of algal meal as feed additive on growth feed efficiency and body composition in the red sea bream. Fish. Sci., 61(1): 25-28.

Norambuena, F., Hermon, K., Skrzypczyk, V., Emery, J. A., Sharon, Y., Beard, A. and Turchini, G. M. 2015. Algae in fish 
feed: Performances and fatty acid metabolism in juvenile Atlantic salmon. PLoS ONE, 10(4): e0124042

Oramary, S. O. M., Koramarky, D. M. I., Salih, S. A. and Mustafa, A. A. 2016. Feeding common carp fish (Cyprinus carpio) on natural foods (algae, phytoplankton, zooplankton and others) on Tigris River in Mosul Dam/Duhok, Kurdistan Region of Iraq. J. Aquac. Res. Dev., 7: 413. doi:10.4172/21559546.1000413

Padhi, S. 1983. Studies on the heterotrophic growth of some blue green algae. Ph. D. Thesis, Berhampur University, India, $342 \mathrm{pp}$.

Patanaik, S., Devi, S. and Padhi, S. 1995. Utilisation of domestic sewage and paper mill effluent for algal biomass production. Ecol. Environ. Cons., 1(1-4): 129-131.

Rausch, T. 1981. The estimation of microalgal protein content and its meaning to evaluation of algal biomass. I. comparison of methods for extracting protein. Hydrobiologia, 78: 237-251.

Samantaray, K. and Mohanty, S. S. 1997. Interaction of dietary level of protein and energy on fingerling snakehead Channa striata. Aquaculture, 156: 241-249.

Satoh, K., Nakagawa, H. and Kasahara, S. 1987. Effects of Ulva meal supplementation on disease resistance of red sea bream. Nippon Suisan Gakk., 53: 1115-1120.

Schroeder, G. L., Wohlfarth, G. W., Alkon, A., Halevy, A. and Krueger, H. 1990. The dominance of algal-based food webs in fish ponds receiving chemical fertilisers plus organic manures. Aquaculture, 86: 219-229.

Steffens, W. 1997. Effects of variation on essential fatty acids in fish feeds on nutritive value of freshwater fish for humans. Aquaculture, 151: 97-119.
Stickney, R. R. and Mc Geachin, R. B. 1983. Responses of Tilapia aurea to semi-purified diets of differing fatty acid composition. In: Proceedings International Symposium on Tilapia in Aquaculture. Nazareth, Israel, p. 346-355.

Stickney, R. R. and Hardy, R. W. 1989. Lipid requirements of some warm water species. Aquaculture, 79: 145-156.

Sumi, K. R. 2011. Effect of different protein levels of fry feed on the production of quality tilapia (Oreochromis niloticus) fry. M.Sc. Thesis. Department of Aquaculture, Bangladesh Agricultural University, $60 \mathrm{pp}$.

Siddika, I., Das, M. and Sumi, K. R. 2012. Effect of isoproteinous feed on growth and survival of tilapia (Oreochromis niloticus) fry. J. Bangladesh Agric. Univ., 10(1): 169-174.

Takeuchi, T., Satoh, S. and Watanabe, T. 1983. Requirement of Tilapia for essential fatty acids. Nippon Suisan Gakk., 49: $1127-1134$

Wee, K. L. and Wang, S. S. 1987. Nutritive value of Leucaena leaf meal in pelleted feed for Nile Tilapia. Aquaculture, 62: $97-108$

Yousif, O. M., Alhadhrami, G. A. and Pessarakli, M. 1994 Evaluation of dehydrated alfalfa and salt bush (Atriplex) leaves in diets for tilapia (Oreochromis aureus L.). Aquaculture, 126: 341-347.

Zamal, H. and Ollevier, F. 1994. Effect of feeding and lack of food on the growth, gross biochemical and fatty acid composition juvenile catfish. J. Fish Biol., 46(3): 404-414.

Zohar, G., Rappaport, U. and Sarig, S. 1985. Intensive culture of Tilapia in concrete tanks. Bamidgeh, 37(4): 103-111. 